Research Article

\title{
Fulminant Type 1 Diabetes in Children: A Multicenter Study in China
}

\author{
Yi Gu, ${ }^{1,2}$ Yi Wang, ${ }^{1}$ Pin Li, ${ }^{3}$ Haiyan Wei, ${ }^{4}$ Linqi Chen, ${ }^{5}$ Qianqi Liu, ${ }^{6}$ Yu Liu, ${ }^{7}$ Qiaozhi Yang, \\ Xinran Cheng, ${ }^{9}$ Lanjie He, ${ }^{10}$ Liya Wei, ${ }^{1}$ Zhiying Zhu, ${ }^{3}$ Yongxing Chen, ${ }^{4}$ Fengyun Wang, ${ }^{5}$ \\ Xing Shi, ${ }^{6}$ Yuxian Cheng, ${ }^{8}$ Yan Wei, ${ }^{9}$ Jianing Yu, ${ }^{10}$ and Chunxiu Gong ${ }^{1}$ \\ ${ }^{1}$ National Key Discipline of Pediatrics, Department of Endocrinology, Genetics and Metabolism, Beijing Children's Hospital, \\ Capital Medical University, National Center for Children's Health, Beijing 100045, China \\ ${ }^{2}$ Department of Microbiology and Immunology, Dartmouth College, Dartmouth-Hitchcock Medical Center, Lebanon, \\ NH 03756, USA \\ ${ }^{3}$ Department of Endocrinology, Children's Hospital of Shanghai, Shanghai Jiao Tong University, Shanghai 200062, China \\ ${ }^{4}$ Department of Endocrinology and Genetic Metabolism, Zhengzhou Children's Hospital, Zhengzhou 450053, China \\ ${ }^{5}$ Department of Endocrinology, Children's Hospital of Soochow University, Suzhou 215003, China \\ ${ }^{6}$ Department of Endocrinology, Nanjing Children's Hospital of Nanjing Medical University, Nanjing 210005, China \\ ${ }^{7}$ Department of Endocrinology, Genetics and Metabolism, The Children's Hospital of Guiyang City, Guiyang 550003, China \\ ${ }^{8}$ Department of Pediatric, Liaocheng Children's Hospital, Shandong 252002, China \\ ${ }^{9}$ The Pediatric Endocrine Department, Chengdu Woman and Children's Center Hospital, Chengdu 610091, China \\ ${ }^{10}$ Department of Pediatric, General Hospital of Ningxia Medical University, Yinchuan 750004, China
}

Correspondence should be addressed to Chunxiu Gong; chunxiugong@sina.com

Received 30 December 2016; Revised 18 April 2017; Accepted 17 May 2017; Published 26 September 2017

Academic Editor: Ruozhi Zhao

Copyright ( 2017 Yi Gu et al. This is an open access article distributed under the Creative Commons Attribution License, which permits unrestricted use, distribution, and reproduction in any medium, provided the original work is properly cited.

Background. To investigate the hospital-based incidence of FT1D in Chinese children and compare the clinical feature with classical T1DM. Methods. A cross-sectional study with sixteen hospitals involved. We obtained 23 FT1D cases as group 1, acute-onset T1DM as group 2, and typical T1DM as group 3. Results. The incidence of FT1D was $1.56 \%$ in 16 participating hospitals. The mean age at the onset of group 1 was $2.00(1.08,6.51)$ years old, much younger than that of group $2(6.11(3.92,9.50))$ and group $3(6.92(4.17,10.03))$. In addition, significant differences were found between three groups: mean BMI and flu-like symptoms with fever and abdominal pain. Follow-up comparison of three groups from Beijing Children's Hospital for at least one year showed that there is no significant difference between the three groups in terms of mean HbA1c levels and insulin injection dosages. Conclusion. FT1D onset age is much younger than that of classical T1D patients. The hospitalbased incidence of FT1D in Chinese children was $1.56 \%$ in all new-onset T1DM. For the diagnosis, making FT1D alone into a subtype within type 1 diabetes may be meaningful. However, for the treatment and prognosis, such classification should not be helpful to the clinic.

\section{Background}

Fulminant type 1 diabetes (FT1D) was first reported by Imagawa et al. in 2000 [1]. Most reported cases are from East Asia and occur during pregnancy or just after delivery $[1,2]$. This type of diabetes is defined as a subtype of type 1 diabetes (T1DM) because it is considered as having a different mechanism from classical T1DM $[1,3,4]$. Since the recognition of FT1D, It had been reported in Korean [5-8], Chinese [9], French [10], and US Hispanic patients [11]. However, there are no data on children (below 15 years old). To clarify the more detailed clinical characteristics and difference between FT1D and classical T1DM in children in China, we performed a multicenter study with 16 hospitals. 


\section{Methods}

2.1. Patients. It is a cross-sectional study with a multicenter design including sixteen hospitals. But in terms of Beijing Children's Hospital, it should be a cohort study. All patients were diagnosed within January 2004 to December 2012. One dedicated doctor was in charge of reviewing all the data and 2 other doctors in auditing according to the same criterion. Finally, we got effective data from nine hospitals. Seven cases (30.43\%) and 16 $(69.57 \%)$ cases were from south and north, respectively (see Figure 1).

Groupings: group 1 included 23 fulminant type 1 diabetes cases. The clinical characteristics of FT1D were [10] (1) remarkably abrupt onset; (2) very short ( $<1$ week) duration of diabetic symptoms (thirst, weight loss, and polyuria); (3) occurrence of diabetic ketosis or ketoacidosis soon (approximately 7 days) after the onset of hyperglycemic symptoms (elevation of urinary and/or serum ketone bodies at first visit); (4) plasma glucose level $\geqq 16.0 \mathrm{mmol} / \mathrm{L}$ ( $\$ 288 \mathrm{mg} / \mathrm{dL}$ ) and $\mathrm{HbAlc}<8.5 \%$ (Japan Diabetes Society value) at first visit; and (5) urinary C-peptide excretion $<10 \mu \mathrm{g} / \mathrm{d}$ or fasting serum C-peptide level $<0.3 \mathrm{ng} / \mathrm{mL} \quad(<0.10 \mathrm{nmol} / \mathrm{L})$ and $<0.5 \mathrm{ng} / \mathrm{mL}(<0.17 \mathrm{nmol} / \mathrm{L})$ after intravenous glucagon (or after meal) load at onset. Other findings in FT1D were (1) flu-like symptoms (fever, upper respiratory symptoms, etc.) or gastrointestinal symptoms (upper abdominal pain, nausea and/or vomiting, etc.). The second group consisted of 182 acute-onset type 1 diabetes cases where (1) patients meet the criteria of the International Diabetes Federation (IDF) and International Society of Pediatric and Adolescent Diabetes (ISPAD) for type 1 diabetes; (2) there is presence of ketosis or ketoacidosis at the onset of diabetes; and (3) the onset of diabetic symptoms was less than 30 days. Group 3 consists of 879 typical type 1 diabetes cases who had diabetic symptoms within 30-100 days. The study program was approved by the ethical committee of the Beijing Children's Hospital.

2.2. Index of Clinical Characteristics and Biochemical Analysis. Data at admission clinically included onset age, sex, hyperglycemic symptom duration, family history of diabetes in first-degree relatives, influenza-like symptoms, blood pressure, and body mass index. Laboratory tests included blood glucose, glycosylated hemoglobin (HbAlc), arterial $\mathrm{pH}$, bicarbonate, $\beta$-hydroxybutyric acid, electrolytes, aspartate aminotransferase, alanine aminotransferase, total cholesterol, and triglyceride. Glutamic acid decarboxylase antibodies (GADAb), insulin autoantibodies (IAA), and islet cell antibodies (ICA) in serum samples were determined with the enzyme-linked immunosorbent assay method. And fasting plasma C-peptide and 2-h postprandial C-peptide levels were determined using the electrochemiluminescence immunoassay method after the resolution of diabetic ketoacidosis.

Some data were missing in other 7 hospitals; we followed up a comparison of three groups of Beijing Children's Hospital.
2.3. Statistical Analysis. Use SPSS17.0 software. Analysis of variance or Kruskal-Wallis H test was used. Group comparisons were done by using least significant difference test. Frequency comparisons were done by using Fisher's exact test. All continuous variables with a normal distribution are expressed as means \pm standard deviation. All tests were twosided, and a $P<0.05$ was required for statistical significance.

\section{Results}

3.1. General Information. There were 23 patients diagnosed with FT1D since 2004-2012, 9 males and 14 females. The incidence of FT1D was $1.56 \%$. Mean age at the onset of group 1 was $2.00(1.08,6.51)$ years old, which was much younger than that of group 2 at $6.11(3.92,9.50)$ years old and group 3 at $6.92(4.17,10.03)$ years old. Significant differences were found between three groups in the mean BMI 16.12 (14.51, 19.55) versus $15.01(13.54,17.29)$ and $14.87(13.61,16.64)$. Abdominal pain was observed in twelve patients $(52.2 \%)$, much more than that in group $2(17.6 \%)$ and group 3 (7.5\%) (see Table 1).

3.2. Biochemical Analysis. Mean plasma glucose in group 1 was higher $(25.10(20.35,30.00))$ than that in group 2 (22.99 (17.99, 30.89)) and group $3(21.78(15.70,28.96))$. Similar results were found in triglycerides: $1.24(0.86,1.59)$ versus $1.45(0.90,2.52)$ and $1.10(0.75,1.83)$. There was also significantly lower arterial blood $\mathrm{pH}$ and lower plasma bicarbonate concentrations $(P=0.001)$ (see Table 1$)$.

For incidence of acute complications of the three groups, a significant difference was found in group 1 in low T3 syndrome, higher than the other two groups $(P=0.018)$. Other acute complications such as serious DKA, HHS, rhabdomyolysis, myocardial damage, and encephaledema were not observed as different between the three groups (see Table 2).

3.3. Follow-Up Results. Follow-up comparison of three groups from Beijing Children's Hospital showed that there is no significant difference in these three groups neither in mean $\mathrm{HbA1c}$ levels nor in insulin injection dosages. Particularly, three groups of patients used minimal insulin injection dosages to maintain plasma glucose level in honeymoon period (see Table 3).

\section{Discussion}

The prevalence of FT1D worldwide is different. $19.4 \%$ of acute-onset type 1 diabetes was revealed in Japan [12] and $7.1 \%$ of newly diagnosed type 1 diabetic patients in Korea [6]. Luo et al. [13] reported 53 cases of FT1D from 24 hospitals nationwide in China, and the percentage of FT1D was $14.9 \%$. However, the patients in the above research were older children and adults. The minimum age of patients enrolled in those researches was 12 years old. The mean age at onset was 35 years in females and 43 years in males; $91.3 \%$ of patients were adults and pregnancy is associated with female fulminant type 1 diabetes [10]. In this study, we collected 23 FT1D patients younger than 15 years who were children from different provinces of China. The 


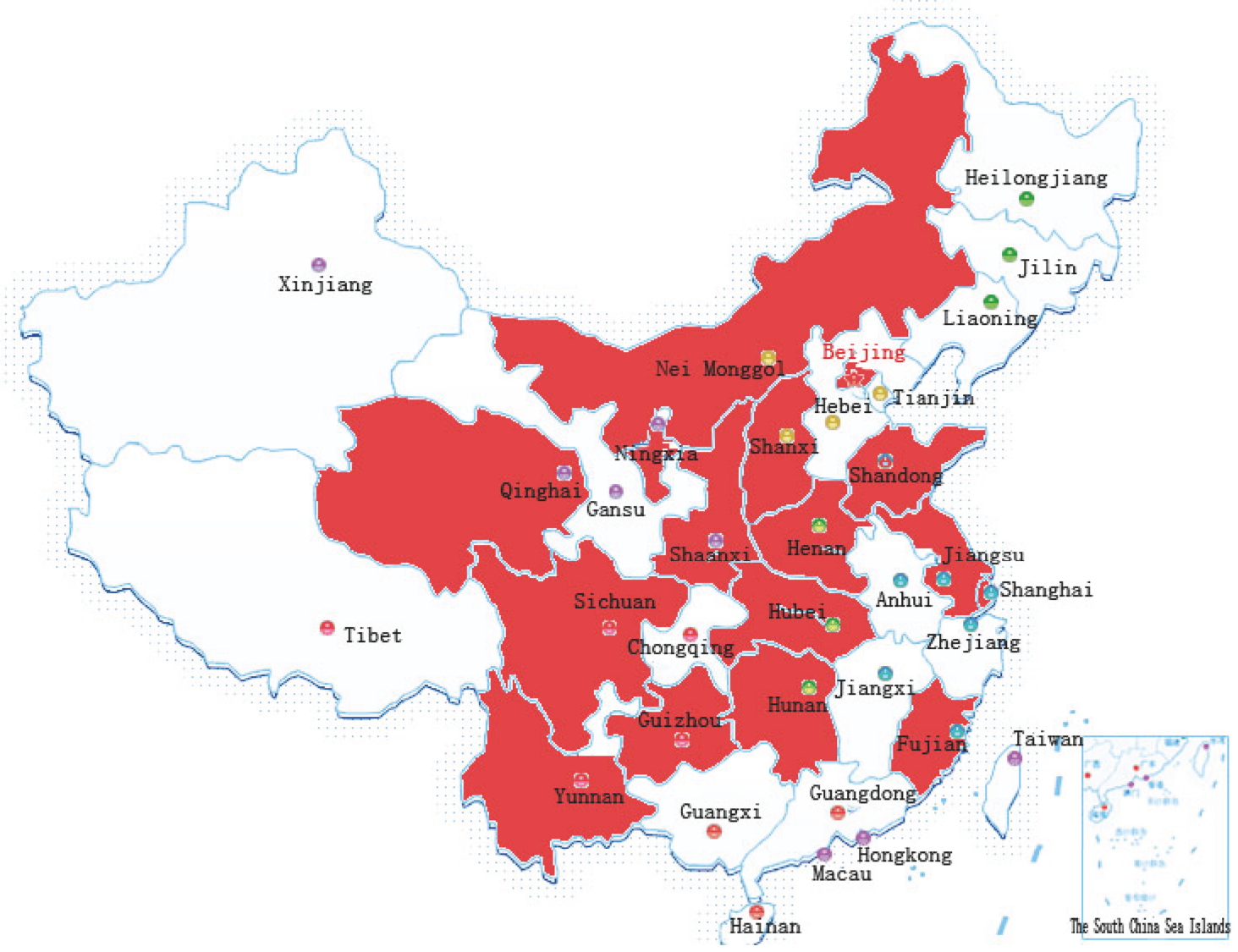

FIgUre 1: The location of each hospital of this study in China.

incidence of FT1D was $1.56 \%$ among all newly T1DM patients, much lower than in adults reported, but it is similar to those Korean studies which reported that the frequency of FT1D was $1.33 \%$ under the age of 16 years [8]. The mean age at onset was 2 years $(1.08,6.51)$. It is quite younger than that in adults, suggesting that the disease occurs in younger age groups of children.

FT1D patients displayed a more diverse clinical manifestation, including flu-like symptoms and gastrointestinal discomforts. In our study, flu-like symptoms and fever were observed in seventeen cases $(73.9 \%)$, much more than acute-onset type 1 diabetes group (10.4\%) and typical type 1 diabetes (4.5\%). Also, abdominal pain was observed in twelve patients $(52.2 \%)$, much more than acute-onset type 1 diabetes group (17.6\%) and typical type 1 diabetes group (7.5\%). These features are similar to those reported in Koreans and Japanese $[6,14]$. Specially, FT1D and acuteonset type 1 diabetes are both acute-onset diseases, but FT1D with flu-like symptoms and abdominal pain was much more frequent than acute-onset T1D. This result indicated that the contribution of viral infection is important to predispose or trigger FT1D. Otherwise, this also seems to explain why the disease occurs in younger age groups of children.

Pathogenesis of FT1D is not clear. Early studies indicated no evidence of islet autoimmunity in FT1D, while recent findings have increasingly suggested that islet autoimmunity is involved in its development [15-17]. Autoantibodies against the $\beta$-cell antigens, such as GAD (two patients), insulin cell autoantibodies (one patient), and islet cell antoantibodies (one patient), were found in these twenty-three FT1D patients. This result accounts for $20 \%$ positive and also has a similar literature report [18-20]. It was given that T-cellmediated autoimmunity played the destruction role of the pancreatic $\beta$-cells $[21,22]$. It is possible that $\beta$-cell-specific Th1 immunity, together with low-grade humoral immune responses, further disposes patients to the development of FT1D. In addition, genetic factors also play a role in the pathogenesis. The HLA class II genes, especially HLA-DQ and HLA-DR, have been associated with a high susceptibility of individuals to autoimmune type 1 diabetes [22]. The DR4DQ4 genes are also associated with the development of FT1D in Japan, and higher frequencies of HLADRB1 ${ }^{*} 0405-\mathrm{DQB1}{ }^{*} 0401$ or HLA-DQA ${ }^{*}$ 0303-DQB1 ${ }^{*} 0401$ and HLA-DQA1 ${ }^{*} 0302-\mathrm{DQB1}{ }^{*} 0303$ haplotypes are observed in Japanese fulminant patients [23-27].

Recently, hypothesis of FT1D is thought to be a coefficient of viral infection and genetic factors [28]. Viral infections will accelerate antiviral immune reactions of CTLA-4, which induce $\beta$-cell death [3]. Moreover, environmental insults such as viral infections are related to alter immune responses in periphery and around the islet [29]. Cytokines such as interleukin and tumor necrosis factor- $\alpha$ recruit additional T cells, macrophages, and NK cells to the islets, and their signaling transductions have direct cytotoxic effects on 
TABLE 1: Clinical features and laboratory tests of the three groups.

\begin{tabular}{|c|c|c|c|c|c|}
\hline & F1TD $(9 / 14)$ & $\begin{array}{c}\text { Acute onset T1DM } \\
(83 / 99)\end{array}$ & $\begin{array}{c}\text { Classical T1DM } \\
(375 / 504)\end{array}$ & $F$ & $P$ \\
\hline Duration of hyperglycemia (days) & $7(2,10)$ & $7(5,8)$ & $15(10,25)$ & 234.736 & $0.000^{\#}$ \\
\hline Age (year) & $2.00(1.08,6.51)$ & $6.11(3.92,9.50)$ & $6.92(4.17,10.03)$ & 14.048 & $0.001^{\#}$ \\
\hline BMI $\left(\mathrm{kg} / \mathrm{m}^{2}\right)$ & $16.12(14.51,19.55)$ & $15.01(13.54,17.29)$ & $14.87(13.61,16.64)$ & 4.621 & 0.099 \\
\hline Family history & 0 & 12 & 32 & - & - \\
\hline Flu-like symptoms & 17 & 19 & 40 & 57.702 & $0.000^{\#}$ \\
\hline Abdominal pain & 12 & 32 & 66 & 29.12 & $0.000^{\#}$ \\
\hline Plasma glucose (mmol/L) & $25.10(20.35,30.00)$ & $22.99(17.99,30.89)$ & $21.78(15.70,28.96)$ & 9.765 & $0.008^{\#}$ \\
\hline $\operatorname{HbA1C}(\%)$ & $7.2 \pm 1.2$ & $11.7 \pm 2.1$ & $11.8 \pm 2.4$ & 43.909 & $0.000^{\#}$ \\
\hline Peptide C (ng/mL) & $0.21(0.09,0.29)$ & $0.31(0.11,0.54)$ & $0.34(0.13,0.58)$ & 8.464 & $0.015^{\#}$ \\
\hline Diastolic blood pressure (mmHg) & $60(60,75)$ & $60(60,70)$ & $60(60,70)$ & 0.619 & 0.734 \\
\hline Systolic pressure (mmHg) & $90(70,103)$ & $100(90,110)$ & $100(90,110)$ & 5.026 & 0.081 \\
\hline $\mathrm{pH}$ & $7.23(6.98,7.34)$ & $7.28(7.14,7.38)$ & $7.32(7.19,7.40)$ & 14.346 & $0.001^{\#}$ \\
\hline $\mathrm{HCO}_{3}(\mathrm{mmol} / \mathrm{L})$ & $8.20(3.10,16.85)$ & $13.10(7.85,18.90)$ & $16.30(8.90,22.10)$ & 16.657 & $0.000^{\#}$ \\
\hline$k(\mathrm{mmol} / \mathrm{L})$ & $4.41(4.00,4.91)$ & $4.10(3.74,4.60)$ & $4.12(3.74,4.51)$ & 3.792 & 0.150 \\
\hline $\mathrm{Na}(\mathrm{mmol} / \mathrm{L})$ & $135.6(131.0,138.0)$ & $135.0(131.1,136.9)$ & $135.0(131.6,138.0)$ & 2.602 & 0.272 \\
\hline $\mathrm{Cl}(\mathrm{mmol} / \mathrm{L})$ & $105.0(98.0,107.0)$ & $103.2(100.0,107.5)$ & $103.2(99.2,106.8)$ & 0.27 & 0.874 \\
\hline Plasma osmolal pressure (Mosm/L) & $284.8(276.7,293.1)$ & $288.8(281.3,297.0)$ & $289.4(282.8,296.6)$ & 2.817 & 0.244 \\
\hline $\mathrm{BUN}(\mathrm{mmol} / \mathrm{L})$ & $4.20(2.70,5.84)$ & $4.48(3.39,6.17)$ & $4.60(3.50,5.88)$ & 1.437 & 0.488 \\
\hline $\mathrm{CHO}(\mathrm{mmol} / \mathrm{L})$ & $3.59(3.24,5.05)$ & $3.99(3.32,4.98)$ & $4.12(3.44,4.90)$ & 1.015 & 0.602 \\
\hline $\mathrm{TG}(\mathrm{mmol} / \mathrm{L})$ & $1.24(0.86,1.59)$ & $1.45(0.90,2.52)$ & $1.10(0.75,1.83)$ & 14.545 & $0.001^{\#}$ \\
\hline AST (U/L) & $30(22,33)$ & $21(15,27)$ & $22(18,29)$ & 8.159 & $0.017^{\#}$ \\
\hline $\operatorname{ALT}(\mathrm{U} / \mathrm{L})$ & $16(12,23)$ & $15(12,18)$ & $15(12,20)$ & 0.808 & 0.668 \\
\hline Insulin dosage of ketoacidosis treatment (IU) & $11.93(7.42,31.35)$ & $13.20(8.40,25.89)$ & $14.43(9.26,28.00)$ & 1.333 & 0.513 \\
\hline Time of ketoacidosis treatment (hour) & $15.0(4.5,21.5)$ & $9.3(6.0,15.6)$ & $10.0(6.0,16.5)$ & 1.043 & 0.594 \\
\hline
\end{tabular}

The statistical method used was analysis of variance. \# represents $P<0.05$.

TABle 2: Acute complications of three groups.

\begin{tabular}{lcccccc}
\hline & Serious DKA & HHS & Low T3 syndrome & Rhabdomyolysis & Myocardial damage & Encephaledema \\
\hline Group 1 & 4 & 0 & 4 & 0 & 1 & 0 \\
Group 2 & 19 & 1 & 10 & 0 & 0 & 30 \\
Group 3 & 76 & 2 & 89 & - & 1.986 & 0 \\
$x^{2}$ & 5.065 & 0 & 8.06 & - & 0.371 \\
$P$ & 0.079 & - & $0.018^{\#}$ & - \\
\hline
\end{tabular}

${ }^{\#}$ There exist differences between group 1 and group 2 and between group 1 and group 3 .

TABLE 3: Follow-up comparison of three groups from Beijing Children's Hospital.

\begin{tabular}{lcccccc}
\hline & \multicolumn{2}{c}{ Course of disease: one month } & \multicolumn{2}{c}{ Course of disease: six months } & \multicolumn{2}{c}{ Course of disease: one year } \\
& HbA1C $(\%)$ & Insulin dosage $(\mathrm{IU} / \mathrm{kg} / \mathrm{d})$ & HbA1C $(\%)$ & Insulin dosage $(\mathrm{IU} / \mathrm{kg} / \mathrm{d})$ & $\mathrm{HbA1C}(\%)$ & Insulin dosage $(\mathrm{IU} / \mathrm{kg} / \mathrm{d})$ \\
\hline Group 1 $(n=5)$ & $7.9 \pm 1.2$ & $0.61 \pm 0.19$ & $7.1 \pm 1.0$ & $0.58 \pm 0.12$ & $7.5 \pm 1.1$ & $0.63 \pm 0.13$ \\
Group 2 $(n=25)$ & $8.2 \pm 1.2$ & $0.51 \pm 0.32$ & $7.8 \pm 2.1$ & $0.58 \pm 0.31$ & $7.5 \pm 1.6$ & $0.49 \pm 0.31$ \\
Group 3 $(n=65)$ & $8.3 \pm 1.4$ & $0.59 \pm 0.35$ & $7.3 \pm 1.6$ & $0.58 \pm 0.33$ & $7.6 \pm 1.7$ & $0.58 \pm 0.35$ \\
$x^{2}$ & 0.170 & 0.555 & 0.889 & 0.000 & 0.013 & 0.726 \\
$P$ & 0.844 & 0.576 & 0.415 & 1.000 & 0.987 & 0.487 \\
\hline
\end{tabular}


$\beta$-cells. The discovery of infiltrating around and in the islet indicates that FT1D may experience a similar pathogenic process as classic type 1 diabetes [30]. In addition to this, Aida et al. [31] studied the in situ status of innate and adaptive immunity of enterovirus-induced FT1D. RIG-I was strongly expressed in $\beta$-cells in pancreas infected with enterovirus. T lymphocyte receptors (TLR3) were expressed in mononuclear cells that infiltrated islets. Interferon- $\alpha$ (IFN- $\alpha$ ) and IFN- $\beta$ were strongly expressed in islet cells. Major histocompatibility complex (MHC) class I, IFN- $\gamma$, interleukin-18, and Cytotoxic C motif ligand 10 were expressed and colocalized in affected islets. Serum levels of IFN- $\gamma$ were markedly increased in patients with T lymphocyte receptor [32]. These findings demonstrate the presence of specific innate immune responses to enterovirus infection in $\mathrm{T}$ lymphocyte receptor. Therefore, the diagnosis of idiopathic diabetes in FT1D is still to be determined. More discussion and accumulation of cases are essential to conclude whether autoimmunity is involved in FT1D.

FT1D patients had significantly lower arterial blood $\mathrm{pH}$ and lower plasma bicarbonate concentrations. Meantime, the serum triglyceride was higher than that in typical type 1 diabetes but not seen in other groups. Otherwise, there is no obvious difference of three groups in acute complications. All these results indicate that FT1D patients display a more diverse clinical manifestation. Significant higher mean BMI was found in FT1D. This related higher BMI phenomenon may be associated with short duration of body weight loss.

There is no significance different in these three groups from Beijing Children's Hospital neither in mean HbAlc levels nor in insulin injection dosages when followed up. Particularly, there is almost the same minimal insulin dosage per day to maintain plasma glucose level in honeymoon period. Therefore, FT1D are similar as acute-onset diabetes and typical type 1 diabetes in insulin treatment and prognosis in children. Indeed, there are some special cases reported which presented another profile, such as Yamashita et al. [33] who reported a woman after acute pancreatitis and FT1D developed simultaneously. She experienced transient complete remission of diabetes and eventually had mild diabetes with non-insulindependency and impaired insulin secretion.

In conclusion, this study showed that the incidence of FT1D below 15 years old was very low, the incidence was $1.56 \%$, and the age of FT1D onset in childhood is much younger. Considering the incidence of this disease and no significant difference of FT1D and type 1 diabetes (mean $\mathrm{HbAlc}$ levels, injection dosages, and minimal insulin injection dosages to maintain plasma glucose level in honeymoon period), we elicit that for the diagnosis, making FT1D alone into a subtype within type 1 diabetes may be meaningful. However, for the treatment and prognosis, such classification should not be helpful to the clinic, while for the limitation of a small size in following up, more follow-up work should be done.

\section{Disclosure}

Yi Gu, Yi Wang, Pin Li, Haiyan Wei, Linqi Chen, Qianqi Liu, Yu Liu, Qiaozhi Yang, Xinran Cheng, and Lanjie He are co- first authors. Liya Wei, Zhiying Zhu, Yongxing Chen, Fengyun Wang, Xing Shi, Yuxian Cheng, Yan Wei, and Jianing $\mathrm{Yu}$ are co-second authors.

\section{Conflicts of Interest}

The authors have no conflict of interest in this study.

\section{Authors' Contributions}

Yi Gu, Yi Wang, Pin Li, Haiyan Wei, Linqi Chen, Qianqi Liu, Yu Liu, Qiaozhi Yang, Xinran Cheng, and Lanjie He contributed equally to this work. Liya Wei, Zhiying Zhu, Yongxing Chen, Fengyun Wang, Xing Shi, Yuxian Cheng, Yan Wei, and Jianing Yu contributed equally to this work.

\section{Acknowledgments}

The authors are grateful to the following pediatrics departments for providing patients with fulminant type 1 diabetes: The Second Xiangya Hospital of Central South University, Inner Monglia Medical University affiliated hospital, First Affiliated Hospital of Kunming Medical University, Xi'an Children's Hospital, Qinghai Woman's and Children's Hospital, Shanxi Children's Hospital, Fuzhou Children's Hospital, and Wuhan Woman and Children Medical Center. This research was supported by two funds: The National Key Research and Development Program of China (the prevalence of diabetes in and nutritional affects on children and adolescents) (no. 2016YFC1305304) and multi-qualified doctors training based on diabetes education and data platform construction (no. 2015400688764J010).

\section{References}

[1] A. Imagawa, T. Hanafusa, J. Miyagawa, and Y. Matsuzawa, "A novel subtype of type 1 diabetes mellitus characterized by a rapid onset and an absence of diabetesrelated antibodies. Osaka IDDM Study Group," The New England Journal of Medicine, vol. 342, no. 5, pp. 301-307, 2000.

[2] E. Kawasaki, T. Maruyama, A. Imagawa et al., "Diagnostic criteria for acute-onset type 1 diabetes mellitus (2012): report of the committee of Japan Diabetes Society on the research of fulminant and acute-onset type 1 diabetes mellitus," Journal of Diabetes Investigation, vol. 5, no. 1, pp. 115-118, 2014.

[3] A. Imagawa and T. Hanafusa, "Fulminant type 1 diabetes-an import subtype in East Asia," Diabetes/Metabolism Research and Reviews, vol. 27, no. 8, pp. 959-964, 2011.

[4] N. Napartivaummuay, T. Suthornthepvarakul, C. Deerochanawong, V. Sarinnapakorn, and S. Niramitmahapanya, "Fulminant type 1 diabetes: the first case report in Thailand," Journal of the Medical Association of Thailand, vol. 96, Supplement 3, pp. s114-s117, 2013.

[5] T. S. Jung, S. I. Chung, M. A. Kim et al., "A Korean patient with fulminant autoantibody-negative type 1 diabetes," Diabetes Care, vol. 27, pp. 3023-3024, 2004.

[6] Y. M. Cho, J. T. Kim, K. S. Ko et al., "Fulminant type 1 diabetes in Korea: high prevalence among patients with adult-onset type 1 diabetes," Diabetologia, vol. 50, pp. 2276-2279, 2007.

[7] N. H. Kim, H. Y. Kim, J. A. Seo et al., "A pooled analysis of 29 patients with fulminant type 1 diabetes in Korea: a comparison 
with a nationwide survey in Japan," Diabetes Research and Clinical Practice, vol. 86, pp. e43-e45, 2009.

[8] M. S. Kim, C. J. Kim, C. W. Ko, P. H. Hwang, and D. Y. Lee, "Fulminant type 1 diabetes mellitus in Korean adolescents," Journal of Pediatric Endocrinology \& Metabolism, vol. 24, no. 9-10, pp. 679-681, 2011.

[9] C. Zheng, Z. Zhou, L. Yang et al., "Fulminant type 1 diabetes mellitus exhibits distinct clinical and autoimmunity features from classical type 1 diabetes mellitus in Chinese," Diabetes/ Metabolism Research and Reviews, vol. 27, pp. 70-78, 2011.

[10] C. Moreau, D. Drui, G. Arnault-Ouary, B. Charbonnel, L. Chaillous, and B. Cariou, "Fulminant type 1 diabetes in Caucasians: a report of three cases," Diabetes \& Metabolism, vol. 34, pp. 529-532, 2008.

[11] R. A. McCauley and X. Wang, "Fulminant type 1 diabetes mellitus-like presentation in a Hispanic woman in the United States," Diabetes \& Metabolism, vol. 37, pp. 356-358, 2011.

[12] A. Imagawa, T. Hanafusa, Y. Uchigata et al., "Fulminant type 1 diabetes-a nationwide survey in Japan," Diabetes Care, vol. 26, pp. 2345-2352, 2003.

[13] S. Luo, Z. Zhang, X. Li et al., "Fulminant type 1 diabetes: a collaborative clinical cases investigation in China," Acta Diabetologica, vol. 50, pp. 53-59, 2013.

[14] R. Mallone, E. Martinuzzi, P. Blancou et al., "CD8+ T-cell responses identify cell autoimmunity in human type 1 diabetes," Diabetes, vol. 56, no. 3, pp. 613-621, 2007.

[15] A. Shimada, J. Morimoto, K. Kodama et al., "T-cell-mediated autoimmunity may be involved in fulminant type 1 diabetes," Diabetes Care, vol. 25, no. 3, pp. 635-636, 2002.

[16] A. Shimada, Y. Oilawa, T. Shigihara, T. Senda, and K. Kodama, "A case of fulminant type 1 diabetes with strong evidence of autoimmunity," Diabetes Care, vol. 25, no. 3, pp. 1482-1483, 2002.

[17] R. Kotani, M. Nagata, A. Imagawa et al., "T lymphocyte response against pancreatic beta cell antigens in fulminant type 1 diabetes," Diabetologia, vol. 47, no. 7, pp. 1285-1291, 2004.

[18] K. Katsumata and K. Katsumata, "A Chinese patient presenting with clinical signs of fulminant type 1 diabetes mellitus," Internal Medicine, vol. 44, no. 9, pp. 967-969, 2005.

[19] T. Iwaoka, "A case of fulminant type 1 diabetes with transiently positive anti-GAD antibodies," Endocrine Journal, vol. 50, no. 2, pp. 225-231, 2003.

[20] Z. Wang, Y. Zheng, Y. Tu, Z. Dai, J. Lin, and Z. Zhou, "Immunological aspects of fulminant type 1 diabetes in Chinese," Journal of Immunology Research, vol. 2016, Article ID 1858202, 6 pages, 2016.

[21] S. Martin, D. Wolf-Eichbanm, G. Duinkerken et al., "Development of type 1 diabetes despite severe hereditary B-cell deficiency," The New England Journal of Medicine, vol. 345, no. 14, pp. 1036-1040, 2001.

[22] B. O. Roep, "The role of T-cells in the pathogenensis of type 1 diabetes: from cause to cure," Diabetologia, vol. 46, no. 3, pp. 305-321, 2003.

[23] T. Nakamura, S. Nagasaka, I. Kusaka, T. Yatagai, J. Yang, and S. Ishibashi, "HLA-DR-DQ haplotype in rapid-onset type 1 diabetes in Japanese," Diabetes Care, vol. 26, no. 5, pp. 16401641, 2003.

[24] S. Murao, H. Makino, Y. Kaino et al., "Differences in the contribution of HLA-DR and DQ haplotypes to susceptibility to adult- and childhood-onset type 1 diabetes in Japanese patients," Diabetes, vol. 53, no. 10, pp. 2684-2690, 2004.
[25] C. Tsutsumi, A. Imagawa, H. Ikegami et al., "Class II HLA genotype in fulminant type 1 diabetes: a nationwide survey with reference to glutamic acid decarboxylase antibodies," Journal of Diabetes Investigation, vol. 3, no. 1, pp. 62-69, 2012.

[26] S. H. Kwak, Y. J. Kim, J. Chae et al., "Association of HLA genotype and fulminant type 1 diabetes in Koreans," Genomics \& Informatics, vol. 13, no. 4, pp. 126-131, 2015.

[27] T. Nishiumi, K. Okamoto, S. Inamoto et al., "Case of fulminant type 1 diabetes mellitus associated with parvovirus B19 infection," Journal of Diabetes Investigation, vol. 5, no. 4, pp. 472-473, 2014.

[28] N. Ohara, M. Kaneko, T. Nishibori et al., "Fulminant type 1 diabetes mellitus associated with Coxsackie virus type A2 infection: a case report and literature review," Internal Medicine, vol. 55, no. 6, pp. 643-646, 2016.

[29] Y. Nishida, K. Aida, M. Kihara, and T. Kobayashi, "Antibodyvalidated proteins in inflamed islets of fulminant type 1 diabetes profiled by laser-capture microdissection followed by mass spectrometry," PLoS One, vol. 9, no. 10, article e107664, 2014.

[30] S. Tanaka, T. Koboyashi, and T. Mototsu, "A novel subtype of type 1 diabetes mellitus," The New England Journal of Medicine, vol. 342, pp. 1835-1183, 2000.

[31] K. Aida, Y. Nishida, S. Tanaka et al., "RIG-I- and MDA5initiated innate immunity linked with adaptive immunity accelerates b-cell death in fulminant type 1 diabetes," Diabetes, vol. 60, pp. 884-889, 2011.

[32] K. Aida, S. Saitoh, Y. Nishida et al., "Distinct cell clusters touching islet cells induce islet cell replication in association with over-expression of regenerating gene (REG) protein in fulminant type 1 diabetes," PLoS One, vol. 9, no. 8, article e105449, 2014.

[33] K. Yamashita, Y. Sato, K. Seki et al., "Fulminant type 1 diabetes with robust recovery of insulin secretion: a case report," Diabetes Research and Clinical Practice, vol. 100, no. 2, pp. e34-e38, 2013. 


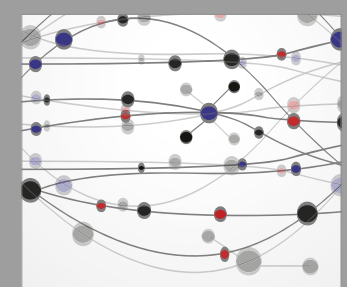

The Scientific World Journal
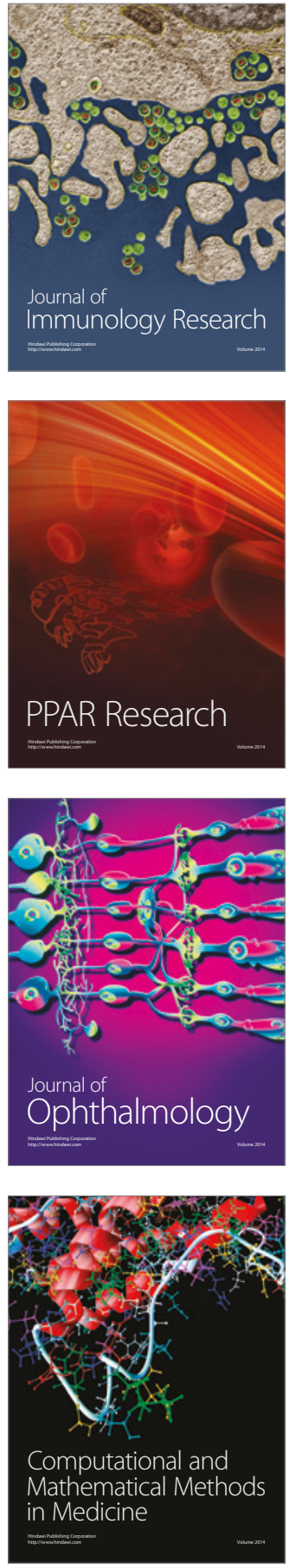

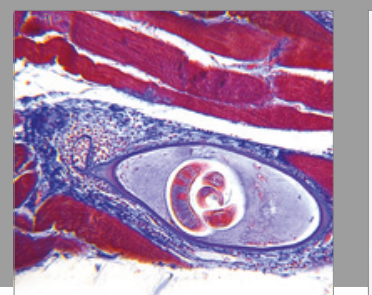

Gastroenterology Research and Practice
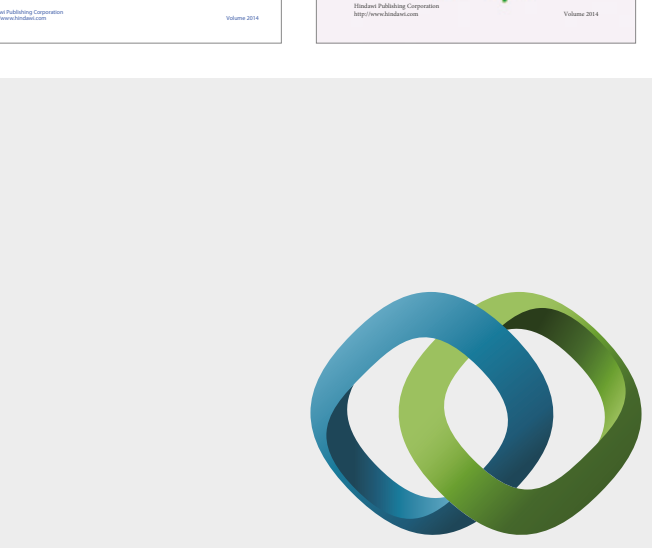

\section{Hindawi}

Submit your manuscripts at

https://www.hindawi.com
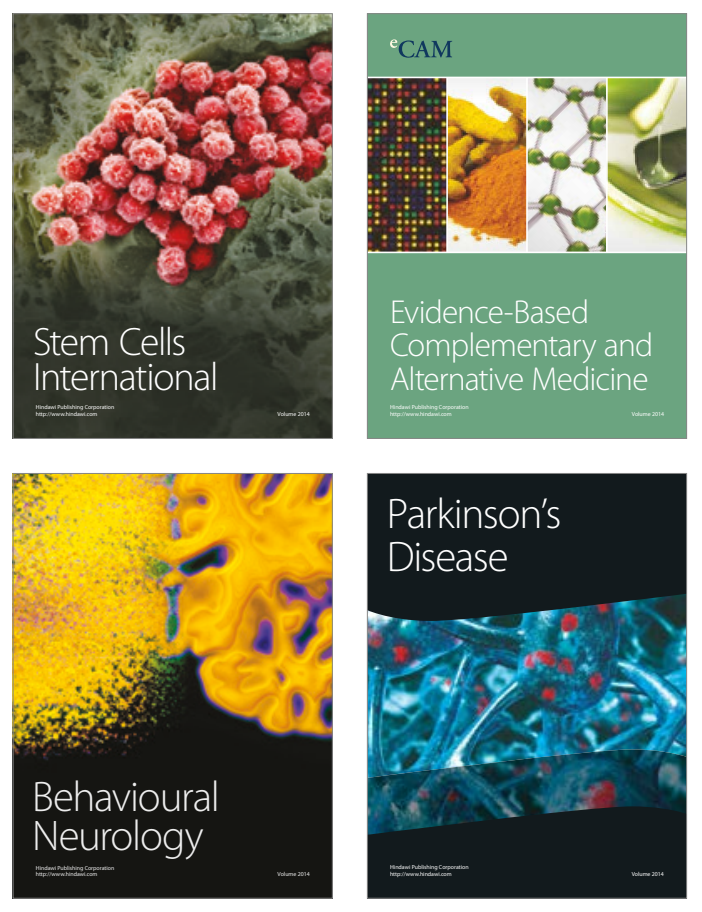
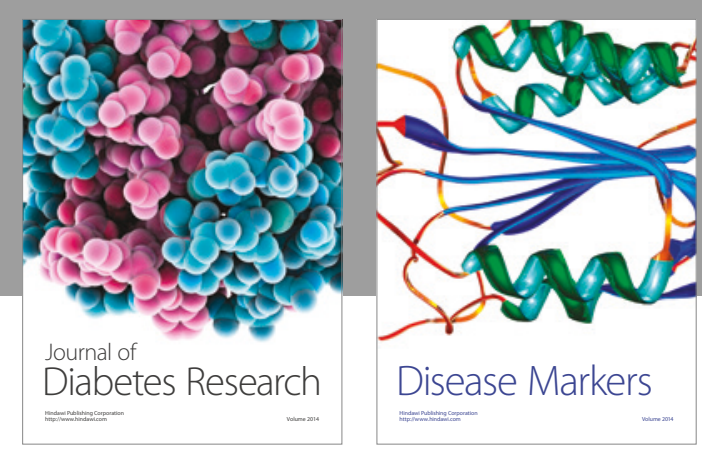

Disease Markers
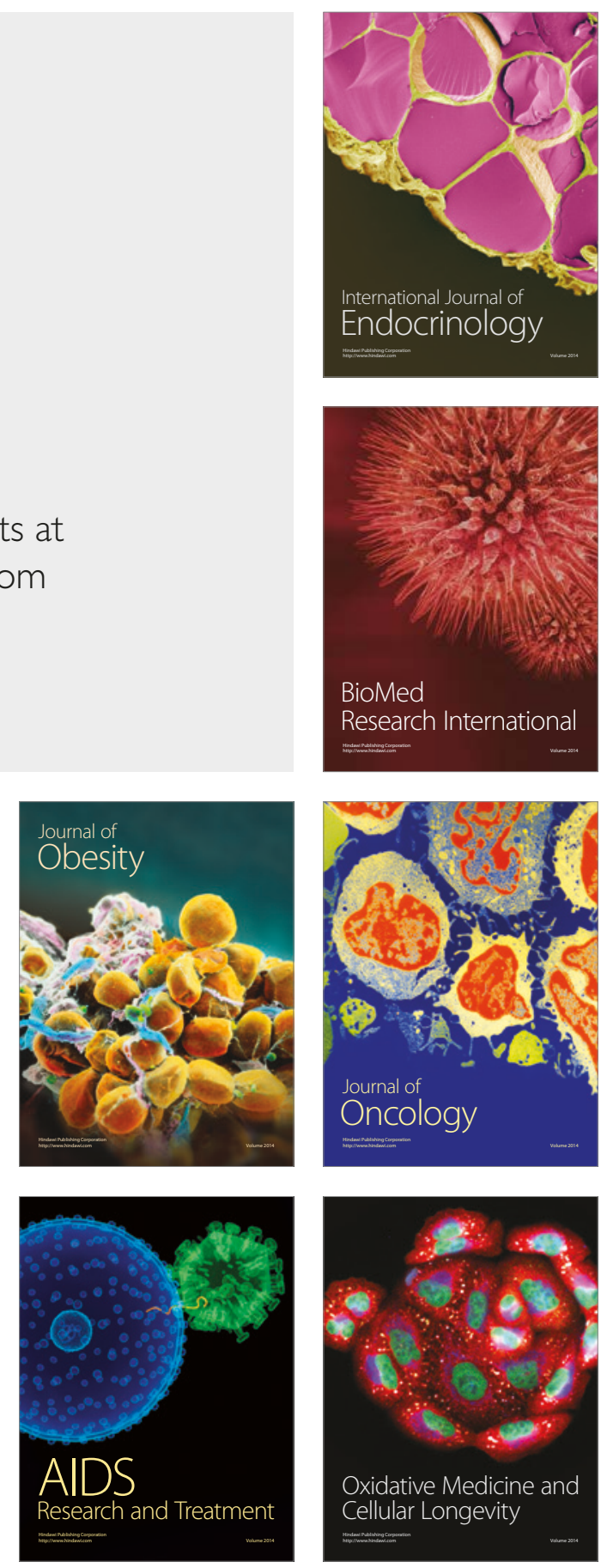